\title{
EGFR Expression Stratifies Oligodendroglioma Behavior
}

\author{
Craig Horbinski, ${ }^{*}$ Jonathan Hobbs, ${ }^{*}$ \\ Kathleen Cieply, ${ }^{\dagger}$ Sanja Dacic, ${ }^{\dagger}$ and \\ Ronald L. Hamilton ${ }^{\dagger}$ \\ From the Department of Pathology, University of Kentucky, \\ Lexington, Kentucky; and the Department of Pathology, \\ University of Pittsburgh, Pittsburgh, Pennsylvania
}

Epidermal growth factor receptor (EGFR) expression and signaling contribute to glioma biological features and, thus, are a target for new drug development. The role, if any, of EGFR in routine surgical neuropathological diagnostics is less clear. Herein, we describe prospective EGFR IHC analysis in an adult cohort comprising $\mathbf{7 5 0}$ infiltrative gliomas. EGFR expression increased with World Health Organization grade but did not significantly differ between grade-matched astrocytic and oligodendroglial tumors. Survival did not significantly differ by EGFR expression among astrocytic tumors adjusted for World Health Organization grade. However, grade II oligodendrogliomas with strong EGFR expression and 1p/19q codeletion showed reduced survival, compared with their codeleted counterparts with weaker EGFR expression. Surprisingly, an inverse phenomenon was found with grade III anaplastic oligodendrogliomas, in which stronger EGFR expression was a favorable marker for survival. Among all gliomas, the likelihood of EGFR amplification, as viewed by fluorescence in situ hybridization, increased with the strength of EGFR expression, and $<1 \%$ of cases with weak or no EGFR immunostaining showed amplification. These data suggest that EGFR IHC is useful in certain circumstances (ie, it may help supplement 1p/19q prognostic information in oligodendroglial tumors and screen out cases that would not benefit from more costly EGFR fluorescence in situ hybridization analysis). (AmJ Pathol 2011, 179:1638-1644; DOI: 10.1016/j.ajpath.2011.06.020)

Epidermal growth factor receptor (EGFR) is a tyrosine kinase that binds to extracellular EGF and dimerizes, thus transducing signal across the cell membrane. This signal elicits a downstream cascade through the mitogen-acti- vated protein kinase/extracellular signal-regulated kinase and phosphatidylinositol 3-kinase/Akt pathways that are generally favorable to growth and cell migration. ${ }^{1}$ Although such signaling is critical for normal organism development, glioma cells frequently use EGFR signaling to promote tumor behavior. Several methods used by gliomas to up-regulate EGFR signaling include overexpression of the receptor via disrupted regulatory feedback, thereby sensitizing the cell to extracellular EGF; overexpression via EGFR gene amplification on 7p12; and a truncation mutation, producing a constitutively active EGFRvIII.2

Given its pro-oncogenic effects, it is not surprising that increased EGFR expression generally correlates with increasing World Health Organization (WHO) grade in gliomas. EGFR amplification is characteristic of approximately $40 \%$ of all glioblastomas (GBMs), and detection of this amplification can be used to identify GBM cells, even in suboptimal tissue biopsy specimens. ${ }^{3}$ The most widely used method of evaluating EGFR copy number is fluorescence in situ hybridization (FISH), in which a fluorophorelabeled DNA probe binds to EGFR DNA and emits a signal. A comparison of the number of EGFR-specific signals with an internal ploidy control (usually a centromeric enumeration probe on chromosome 7 labeled with a different fluorophore) yields the EGFR copy number in a given DAPI-counterstained nucleus; by convention, EGFR amplification is diagnosed when the EGFR/centromeric enumeration probe for chromosome 7 (CEP7) ratio is $>2.4$

Testing for EGFR amplification serves mainly as a refinement of diagnosis because its presence in a brain biopsy specimen strongly suggests that the tumor is a GBM, even if the histological and/or radiological features do not match up. Furthermore, a tumor that has oligodendroglial morphological features yet shows EGFR amplification may be a

Supported in part by a University of Kentucky College of Medicine Physician-Scientist Fellowship (C.H.).

Accepted for publication June 3, 2011

Supplemental material for this article can be found at http://ajp. amjpathol.org or at doi: 10.1016/j.ajpath.2011.06.020

Address reprint requests to Craig Horbinski, M.D., Ph.D., Department of Pathology, University of Kentucky, MS-112, 800 Rose St., Lexington, KY 40536. E-mail: craig.horbinski@uky.edu. 
small-cell variant of GBM. ${ }^{5,6}$ Testing for EGFR expression and/or amplification may also prove useful from a therapeutics perspective, assuming that an anti-EGFR drug can be developed that has significant therapeutic effects. ${ }^{7,8}$

Several retrospective studies ${ }^{9-14}$ have tested the correlation between EGFR expression in WHO gradeadjusted gliomas and patient survival, with conflicting data. Herein, we describe the results of EGFR expression analysis from a prospective cohort of 750 infiltrative gliomas covering WHO grades II to IV, including its ability to predict EGFR amplification and its correlation with patient survival.

\section{Materials and Methods}

\section{Cohort}

From January 24, 2002 to August 11, 2010, biopsy material from 750 gliomas in the adult population $(\geq 18$ years) was analyzed at initial diagnosis for EGFR and $1 p / 19 q$ status at the University of Pittsburgh Medical Center (Pittsburgh, PA), as described later. Cases of recurrent or treated gliomas were excluded. Diagnoses were rendered according to WHO criteria at initial biopsy. Hybrid diagnoses, such as oligoastrocytoma, were avoided. Survival, marked from the original biopsy, was determined using the Social Security Death Index. Specific information about treatment regimens in each patient was not available.

\section{EGFR IHC Analysis}

EGFR immunohistochemistry (IHC) was performed on sections (5- $\mu \mathrm{m}$ thick) obtained from paraffin-embedded material, using EGFR primary antibody (Ventana 7902988/3C6/prediluted; Ventana Medical Systems, Oro Valley, AZ). The antibody labeling was performed using the avidin-biotin complex method and visualized using a horseradish peroxidase enzyme label and 2'-diaminobenzamide (Dako, Carpinteria, CA) as the substrate chromogen (brown).

EGFR IHC analysis was based on the following semiquantitative scale: 0 , negative; 1 , weak; 2 , moderate; and 3 , strong. The score was predicated on the strongest area of the tumor.

\section{FISH Analysis}

FISH analyses for EGFR and 1p/19q were performed as previously described. ${ }^{15}$ Briefly, formalin-fixed, paraffinembedded tissues were mounted and serially divided into sections (5- $\mu \mathrm{m}$ thick). An H\&E section was used by the pathologist to determine the area of the tissue to be targeted for analysis. FISH slides were deparaffinized in xylene twice for 10 minutes, dehydrated twice with 100\% ethanol, and then pretreated using the Vysis Paraffin Pretreatment Kit (Abbott Laboratories, Des Plaines, IL). Slides were digested for 18 minutes in protease solution $(0.5 \mathrm{mg} / \mathrm{mL})$ at $37^{\circ} \mathrm{C}$. FISH was performed using probes for EGFR (7p12)/CEP7, 1p36/1q25, and 19q13/19p13
(Abbott Molecular, Des Plaines, IL). The target slide and probe were codenatured at $95^{\circ} \mathrm{C}$ for 8 minutes and incubated overnight at $37^{\circ} \mathrm{C}$ in a humidified chamber. Posthybridization washes were performed using two times standard saline citrate/0.3\% Igepal (Sigma, St Louis, MO) at $72^{\circ} \mathrm{C}$ for 2 minutes. Slides were air dried in the dark and counterstained with DAPI (Abbott Molecular). Analysis was performed using a Nikon Optiphot-2 (Nikon, Inc., Melville, NY) and Quips Genetic Workstation (Abbott Laboratories, Des Plaines, IL) equipped with a Chroma Technology filter (Bellows Falls, VT) set with single-band exciters for SpectrumOrange, fluorescein isothiocyanate, and DAPI (UV, $360 \mathrm{~nm}$ ).

In each case, a minimum of 60 tumor cells were scored. EGFR amplification was defined as an EGFR/ CEP7 ratio $\geq 2.0$. $1 \mathrm{p} / 19 \mathrm{q}$ codeletion was determined when both $1 \mathrm{p} 36 / 1 \mathrm{q} 25$ and $19 \mathrm{q} 13 / 19 p 13$ signal ratios were $<0.85$ in $>20 \%$ of tumor cells. These cutoff points were derived using nonneoplastic autopsy brain tissue as controls.

\section{Statistical Analysis}

Survival rates were compared via log-rank tests on $\mathrm{Ka}$ plan-Meier curves. Correlations between EGFR IHC and EGFR amplification were performed via nonparametric Spearman rank correlation. EGFR IHC scores were compared between diagnostic groups, and EGFR amplification status was compared via Kruskal-Wallis analysis with Dunn's post hoc test. The likelihood of EGFR amplification or $1 p / 19 q$ codeletion, based on $\mathrm{IHC}$ score, was determined using the Fisher's exact test. All analyses were performed using GraphPad software (GraphPad Software, Inc., La Jolla, CA); differences were considered significant if $P<0.05$.

\section{Results}

\section{Cohort Characteristics}

The prospective cohort comprised 750 individuals with infiltrative gliomas (Table 1). Overall, $58.7 \%$ were male, the male/female ratio was 1.4:1, and the median age was 59 (range, 18 to 94) years. Tumor categorization was based on the original diagnosis rendered at biopsy. Nearly $70 \%$ of all tumors were GBM, followed by grade II oligodendroglioma (11.2\%), grade III anaplastic astrocytoma $(8.5 \%)$, grade II diffuse astrocytoma $(8.1 \%)$, and grade III anaplastic oligodendroglioma (4.5\%). All of these parameters are generally consistent with prior population-based data. ${ }^{16}$

Of the 750 patients, $466(62.1 \%)$ were dead at the time of study. The median follow-up interval for all patients was 330 days (range, 3 days to 8.8 years). Of those patients who died, the median survival was 219 days (range, 3 days to 6.4 years).

\section{EGFR IHC and Gene Amplification}

EGFR IHC analysis was performed at original diagnosis on all cases (see Materials and Methods). Cases were 
Table 1. Analysis of EGFR Expression in Gliomas within the Study Cohort

\begin{tabular}{|c|c|c|c|c|c|c|c|c|c|c|}
\hline \multirow{2}{*}{$\begin{array}{c}\text { WHO } \\
\text { diagnosis }\end{array}$} & \multirow{2}{*}{$\begin{array}{c}\text { All } \\
\text { individuals }\end{array}$} & \multirow[b]{2}{*}{ Males } & \multirow[b]{2}{*}{ Females } & \multirow{2}{*}{$\begin{array}{c}\text { Median } \\
\text { (range) } \\
\text { age } \\
\text { (years) }\end{array}$} & \multirow{2}{*}{$\begin{array}{c}\text { Mean } \pm \\
\text { SEM } \\
\text { EGFR IHC } \\
\text { score }\end{array}$} & \multicolumn{4}{|c|}{ EGFR data } & \multirow{2}{*}{$\begin{array}{c}\text { EGFR } \\
\text { amplification }\end{array}$} \\
\hline & & & & & & Negative & Weak & Moderate & Strong & \\
\hline \multicolumn{11}{|c|}{ Astrocytoma } \\
\hline Grade II & $61(8.1)$ & $31(50.8)$ & $30(49.2)$ & $48(20-81)$ & $1.5 \pm 0.1$ & $4(6.6)$ & $30(49.2)$ & $19(31.1)$ & $8(13.1)$ & $3(4.9)$ \\
\hline Grade III & $64(8.5)$ & $39(60.9)$ & 25 (39.1) & $56(22-94)$ & $2.2 \pm 0.1$ & $1(1.6)$ & $14(21.9)$ & 21 (32.8) & $28(43.8)$ & $17(26.6)$ \\
\hline GBM & $507(67.6)$ & $301(59.4)$ & $206(40.6)$ & $63(18-89)$ & $2.4 \pm 0.04$ & $12(2.4)$ & $64(12.6)$ & $140(27.6)$ & $291(57.4)$ & $224(44.2)$ \\
\hline \multicolumn{11}{|c|}{ Oligodendroglioma } \\
\hline Grade II & $84(11.2)$ & $46(54.8)$ & 38 (45.2) & 42 (19-78) & $1.8 \pm 0.09$ & $7(8.3)$ & 18 (21.4) & $42(50.0)$ & $17(20.2)$ & $1(1.2)$ \\
\hline Grade III & $34(4.5)$ & $23(67.6)$ & $11(32.4)$ & $48(32-80)$ & $2.3 \pm 0.1$ & $0(0)$ & 7 (20.6) & $10(29.4)$ & $17(50.0)$ & $2(5.9)$ \\
\hline Total & $750(100.0)$ & $440(58.7)$ & $310(41.3)$ & $59(18-94)$ & $2.2 \pm 0.03$ & $24(3.2)$ & $133(17.7)$ & $232(30.9)$ & $361(48.1)$ & $247(32.9)$ \\
\hline
\end{tabular}

Data are given as number (percentage) unless otherwise indicated. A total of 750 cases of diffusely infiltrative glioma were analyzed prospectively for EGFR expression, EGFR amplification, and 1p/19q codeletion (see Materials and Methods).

prospectively scored using a semiquantitative method: 0 , negative; 1, weak; 2, moderate; and 3, strong (Figure 1, A-D). EGFR expression was lower in grade II gliomas compared with grade III to IV gliomas (overall $P<$ 0.0001 , Figure 1E). There was no significant difference in expression after adjusting for grade and histotype (ie, astrocytoma versus oligodendroglioma).

In all gliomas, there was a strong correlation between the degree of EGFR expression and EGFR gene amplifi-
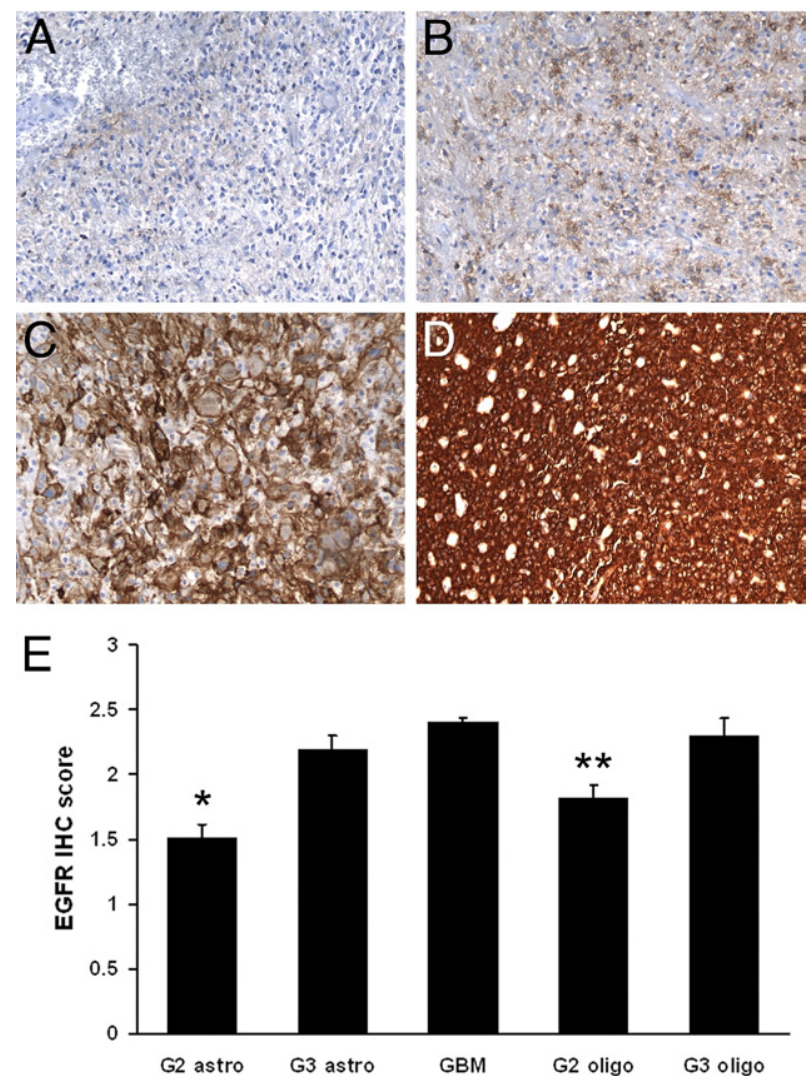

Figure 1. EGFR IHC in gliomas. IHC stains for EGFR were semiquantitatively scored on a scale, as follows: 0, negative (A); 1 , weak (B); 2, moderate (C); and 3, strong (D). E: EGFR staining is generally weaker in grade II gliomas and equally strong among grade III to IV gliomas. ${ }^{*} P<0.001$ versus grade III astrocytoma (G3 astro) and GBM; ${ }^{* *} P<0.001$ versus GBM. Original magnification: $\times 200(\mathbf{A}-\mathbf{E})$ cation detected by FISH (two-tailed $P<0.0001$ by nonparametric Spearman rank correlation, Figure 2A). Specifically, none of the 24 gliomas with completely negative EGFR IHC had EGFR amplification, and only $1(0.75 \%)$ of the 133 cases with weak EGFR IHC showed amplification; that case was a GBM histologically (not shown). There was a sharp increase in amplification frequency to $10.8 \% \pm 2.0 \%$ ( \pm SEM) with a moderate $\mathrm{IHC}$ score and a nearly sixfold increase to $61.2 \% \pm 2.6 \%$ ( \pm SEM) when there was strong EGFR expression.

Classifying the cohort by $\mathrm{WHO}$ diagnostic category also revealed a highly significant difference in the proportion of cases with EGFR amplification, according to EGFR IHC score ( $P<0.0001$, Figure 2B). An average of $9.7 \%$ of all moderately staining gliomas had EGFR amplification. Interestingly, the frequency did not vary between
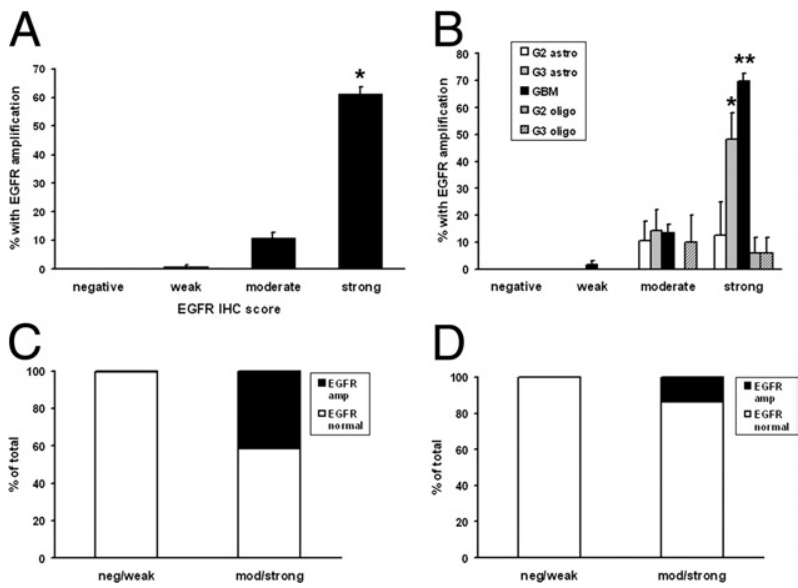

Figure 2. Correlation between EGFR expression and gene amplification. A total of 750 WHO grade II to IV gliomas were prospectively immunostained for EGFR and tested for gene amplification by FISH (see Materials and Methods). A: In all gliomas, the rate of EGFR amplification increased with increasing EGFR staining intensity. ${ }^{*} P<0.001$ versus all other groups. B: Adjusting for WHO grade and morphologic subtype, the highest rates of EGFR amplification were seen in grades III and IV astrocytomas with strong EGFR expression. ${ }^{*} P<0.05$ versus all negative and weak tumors; ${ }^{* *} P<0.05$ versus all but strong grade III astrocytoma (G3 astro). C: The risk of EGFR amplification (amp) was nearly nonexistent in all gliomas with negative (neg) or weak EGFR immunostaining, including GBMs. $P<0.0001$. D: Specifically in non-GBM gliomas (in which EGFR amplification might change the diagnosis), there was a greatly increased risk of amplification if the tumor was moderately (mod) or strongly immunopositive for EGFR, although the rate was still only $14 \% . P<0.0001$ 
Table 2. Ability of Moderate-to-Strong EGFR Expression to Predict EGFR Amplification in Diffusely Infiltrative Gliomas

\begin{tabular}{cccc}
\hline Diagnostic parameter & All gliomas & Non-GBM gliomas & GBM only \\
\hline Sensitivity & $1.0(0.98-1.0)$ & $1.0(0.85-1.0)$ & $1.0(0.98-1.0)$ \\
Specificity & $0.39(0.34-0.44)$ & $0.37(0.30-0.44)$ & $0.27(0.21-0.32)$ \\
PPV & $0.59(0.54-0.62)$ & $0.14(0.088-0.20)$ & $0.52(0.47-0.57)$ \\
NPV & $0.99(0.97-1.0)$ & $1.0(0.96-1.0)$ & $0.99(0.93-1.0)$ \\
\hline
\end{tabular}

The $95 \%$ Cls are given in parentheses. Moderate-to-strong EGFR IHC immunoreactivity (see Figure 1) is extremely sensitive and has a uniformly high NPV, but its specificity and PPV vary whether the focus is on all gliomas, non-GBM gliomas, or GBM only.

NPV, negative predictive value; PPV, positive predictive value.

diagnostic categories, with the exception of grade II oligodendrogliomas, where 0 of 42 moderately staining tumors had EGFR amplification. Strongly staining anaplastic astrocytomas and GBMs had the highest rates of EGFR amplification at $48.1 \%$ and $69.9 \%$, respectively.

Evaluating EGFR IHC as a diagnostic test showed that pooling moderate to strongly positive immunostaining among all gliomas is highly sensitive and has a robust negative predictive value for EGFR amplification (1.0 and 0.99, respectively; Figure 2, C and D; Table 2). Because many cases had moderate or strong immunoreactivity yet were still not EGFR amplified, the specificity and positive predictive values were lower (0.39 and 0.59 , respectively). In particular, moderate or strong EGFR IHC had a relatively low positive predictive value (0.14) in non-GBM gliomas.

\section{Association of EGFR Expression with Survival}

Pooling all gliomas together, overall survival generally decreased with increasing expression of EGFR IHC $(P<$ 0.0001, Figure 3A, Table 3). Yet, adjusting for WHO grade eliminated the survival impact of EGFR expression in astrocytic tumors (Figure 3, B-D). The only exception was in the case of GBM, in which survival was slightly worse in moderate EGFR-expressing GBMs compared with strong expressers (median survival, 0.57 versus 0.80 years; $P=0.03$ ) (Figure 3D, Table 3 ). Also, in the GBM subset, the median survival of EGFR-negative GBMs (1.2 years) was longer than that seen in weak (0.68 years), moderate (0.57 years), or strong (0.80 years) EGFR expressers, although none of these differences were statistically significant. Too few cases of grades II and III astrocytomas were EGFR negative to produce reliable results (Figure 3, B and C; Table 1).

Interestingly, the situation was different in oligodendroglial tumors. Grade II oligodendrogliomas with strong EGFR expression fared worse than those with weaker EGFR staining (Figure 4A). Specifically, the median survival of grade II oligodendrogliomas with strong EGFR expression was 4.3 years, in contrast to the undefined median survival times of grade II oligodendrogliomas with lesser degrees of EGFR expression $(P=0.02$, Table 3). This was independent of $1 p / 19 q$ codeletion because grade II oligodendrogliomas with $1 p / 19 q$ codeletion and strong EGFR expression still had worse overall survival compared with $1 p / 19 q$-codeleted tumors with weaker EGFR expression ( $P=0.004$, Figure 4B). Furthermore, there was no significant difference in the likelihood of $1 \mathrm{p} / 19 \mathrm{q}$ codeletion according to EGFR expression; 14 (82.4\%) of 17 oligodendrogliomas with strong EGFR staining had $1 \mathrm{p} / 19 \mathrm{q}$ codeletion, whereas $44(66.7 \%)$ of 66 oligodendrogliomas with negative, weak, or moderate EGFR staining had $1 p / 19 q$ codeletion $(P=0.25$ via Fisher's exact test, Figure 4C).

The situation was completely reversed in grade III anaplastic oligodendrogliomas, in which tumors with weak EGFR expression had a shorter median survival (1.1 years) compared with moderate (undefined) or strong
A

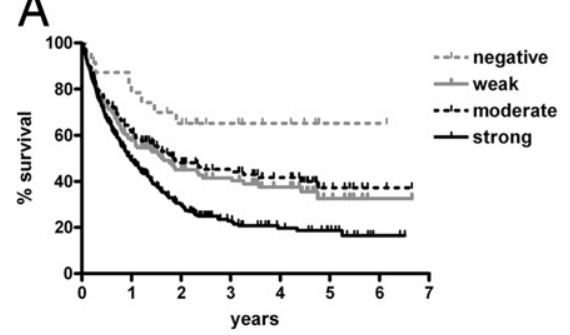

D

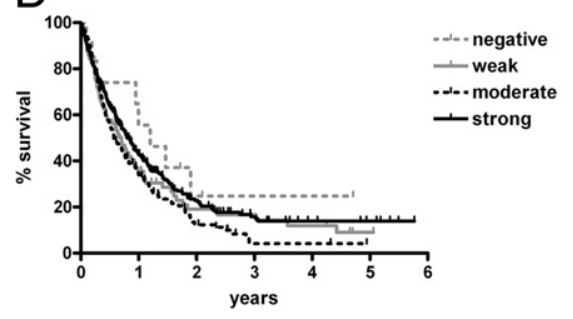

B
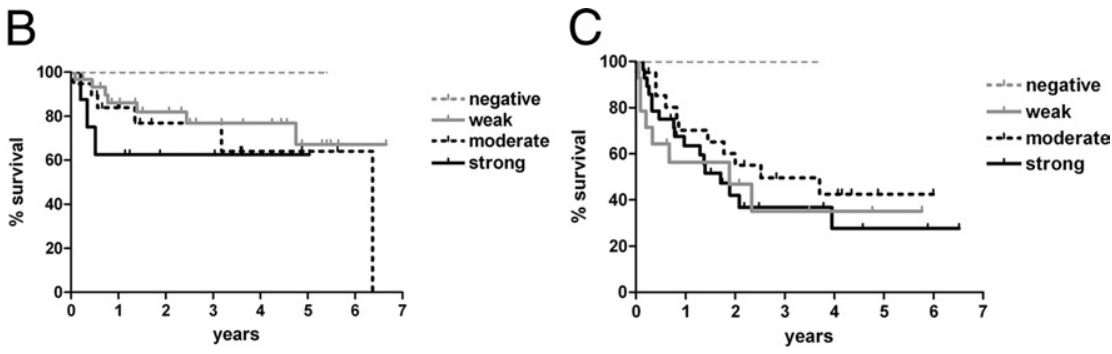

Figure 3. Glioma survival by EGFR IHC scores. A: WHO grade II to IV gliomas were segregated according to strength of EGFR IHC. In general, increased EGFR correlated with shorter overall survival. $P<0.0001$. However, adjusting for WHO grade in astrocytic tumors eliminated any significant association between EGFR expression and survival in grades II (B), III (C), and IV (D) astrocytomas. See Table 3 for specific intergroup $P$ values. Although the survival curves in EGFR-negative grades II and III astrocytomas appeared favorable, there were not enough cases of each to generate statistical significance (Table 1). 
1642 Horbinski et al

AJP October 2011, Vol. 179, No. 4

Table 3. Median Survival according to EGFR IHC Status

\begin{tabular}{|c|c|c|c|c|c|c|c|c|c|c|c|}
\hline \multirow[b]{2}{*}{$\begin{array}{l}\text { WHO } \\
\text { group }\end{array}$} & \multicolumn{4}{|c|}{ Median survival by EGFR IHC (years) } & \multirow[b]{2}{*}{ Overall $P$} & \multicolumn{6}{|c|}{$P$ values between EGFR IHC subgroups } \\
\hline & Neg & Weak & Mod & Strong & & $\begin{array}{c}\text { Neg vs } \\
\text { weak }\end{array}$ & $\begin{array}{l}\text { Neg vs } \\
\text { mod }\end{array}$ & $\begin{array}{l}\text { Neg vs } \\
\text { strong }\end{array}$ & $\begin{array}{c}\text { Weak } \\
\text { vs mod }\end{array}$ & $\begin{array}{c}\text { Weak vs } \\
\text { strong }\end{array}$ & $\begin{array}{l}\text { Mod vs } \\
\text { strong }\end{array}$ \\
\hline All gliomas & Undefined & 1.6 & 1.9 & 0.99 & $<0.0001^{*}$ & 0.029 & 0.058 & $0.0003^{*}$ & 0.50 & $0.0032^{*}$ & $<0.0001^{*}$ \\
\hline \multicolumn{12}{|c|}{ Astrocytoma } \\
\hline Grade II & Undefined & Undefined & 6.37 & Undefined & 0.32 & 0.25 & 0.21 & 0.14 & 0.45 & 0.25 & 0.40 \\
\hline Grade III & Undefined & 1.9 & 2.5 & 1.7 & 0.54 & 0.33 & 0.41 & 0.33 & 0.36 & 0.88 & 0.29 \\
\hline GBM & 1.2 & 0.68 & 0.57 & 0.80 & 0.097 & 0.23 & 0.12 & 0.39 & 0.43 & 0.36 & $0.025^{\star}$ \\
\hline \multicolumn{12}{|c|}{ Oligodendroglioma } \\
\hline Grade II & Undefined & Undefined & Undefined & 4.3 & $0.02^{*}$ & 0.37 & 0.40 & $0.050^{*}$ & 0.71 & 0.084 & $0.013^{*}$ \\
\hline Grade III & NA & 1.1 & Undefined & 2.7 & $0.03^{*}$ & NA & NA & NA & $0.025^{*}$ & $0.016^{*}$ & 0.79 \\
\hline
\end{tabular}

A total of 750 diffusely infiltrative astrocytomas and oligodendrogliomas, WHO grades II to IV, were prospectively analyzed for EGFR expression via IHC semiquantification (see Materials and Methods). Median survival after diagnosis was determined by Kaplan-Meier curves, with statistical significance calculated via log-rank tests.

*Significant correlations.

mod, moderate; NA, not available (no grade III oligodendrogliomas were immunonegative for EGFR); neg, negative.

(2.7 years) EGFR expressers ( $P=0.03$, Figure 5A, Table 3). Stratification according to $1 p / 19 q$ status showed that the only subgroup with relatively favorable survival was tumors with both $1 \mathrm{p} / 19 \mathrm{q}$ codeletion and moderate-tostrong EGFR expression ( $P=0.04$, Figure $5 \mathrm{~B})$. Three of the 27 EGFR moderate-to-strong anaplastic oligodendrogliomas had intact $1 \mathrm{p} / 19 \mathrm{q}$, whereas two of seven cases with weak EGFR staining were $1 \mathrm{p} / 19 \mathrm{q}$ intact $(P=0.27$ by Fisher's exact test, Figure 5C).

\section{Discussion}

Efforts to identify biomarkers of outcome in gliomas have been ongoing for several decades. Such biomarkers have greatly increased in number and power since the development of advanced molecular diagnostic techniques. Even for those markers that have repeatedly proved to be useful from a diagnostic and/or therapeutic perspective, an additional dimension of cost-effectiveness is becoming prominent. Thus, less expensive and more rapid screening tools for key molecular alterations are desirable.

Smaller retrospective studies ${ }^{12,17-19}$ have previously suggested that EGFR IHC often correlates with EGFR gene amplification, although there is less of an association between EGFR expression and amplification in pediatric high-grade gliomas; many will show at least some expression, yet amplification is rare. ${ }^{20}$ In the current large prospective cohort of adult gliomas, EGFR IHC at diagnosis was a highly sensitive, although not specific, marker for EGFR amplification. In particular, cases that are negative or weak for EGFR expression are extremely unlikely to have EGFR amplification and, thus, probably do not require more laborious and expensive FISH testing.

Furthermore, because the likelihood of EGFR amplification increases with WHO glioma grade and is most common in GBM (Table 1), it is not surprising that the positive predictive value of EGFR $\mathrm{IHC}$ is higher when applied specifically to higher-grade tumors. Grades III and IV astrocytomas had the highest rate of EGFR amplification and the best correlation between strong EGFR $\mathrm{IHC}$ and amplification (Figure 2B). Between 5\% and 15\% of grade II astrocytomas and grade II to III oligodendrogliomas with either moderate or strong EGFR $I H C$ showed EGFR amplification. Because EGFR amplification is generally considered most consistent with a de novo GBM, ${ }^{16,21}$ and oligolike tumors with EGFR amplification are more likely to be called small-cell GBMs, ${ }^{5,6}$ it might be worthwhile to follow up with FISH on such cases because the diagnosis could be completely altered. This is especially relevant if there is a question about whether the biopsy material fully represents the histologic characteristics of the entire tumor. ${ }^{3}$ The median survival of
A

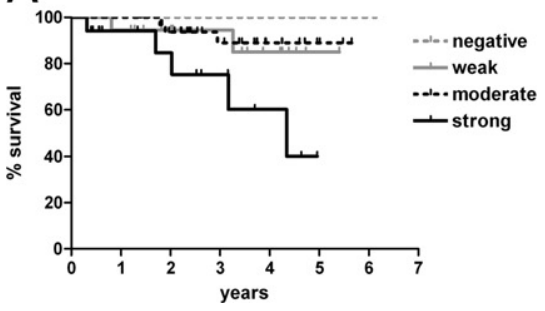

B

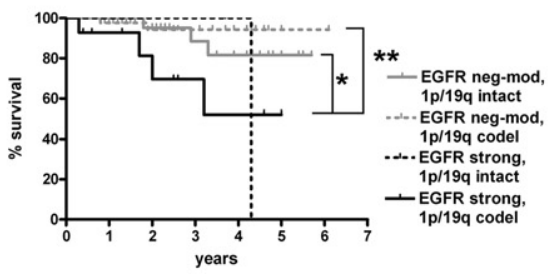

C

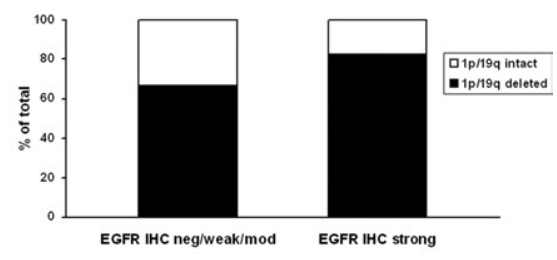

Figure 4. Survival by EGFR IHC in grade II oligodendrogliomas. A: Grade II oligodendrogliomas show progressively worse survival with increasing EGFR IHC intensity. $P=0.02$. See Table 3 for specific intergroup $P$ values. B: Adjusting for $1 \mathrm{p} / 19 \mathrm{q}$ codeletion required combining cases that were EGFR IHC negative (neg), weak, and moderate (mod) into one group (neg-mod), with strong EGFR cases as a separate category, then segregating according to $1 \mathrm{p} / 19 \mathrm{q}$ status. Even cases with $1 \mathrm{p} / 19 \mathrm{q}$ codeletion showed worse survival if EGFR expression was strong. $P=0.02$ overall, ${ }^{*} P=0.05$, and ${ }^{* *} P=0.004$. C: There was no difference in the likelihood of $1 \mathrm{p} / 19 \mathrm{q}$ codeletion between oligodendrogliomas that were strong for EGFR versus those with weaker staining. $P=0.25$. 
A

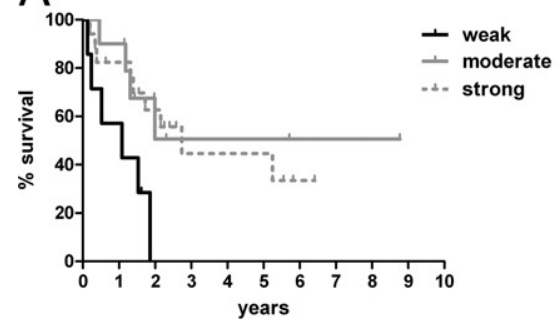

B

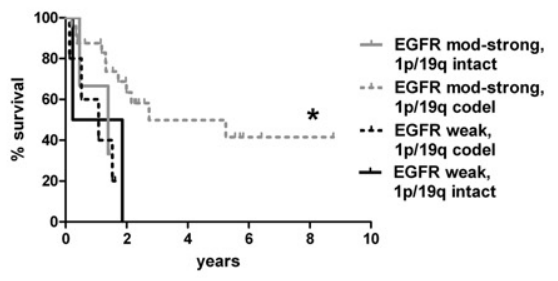

C

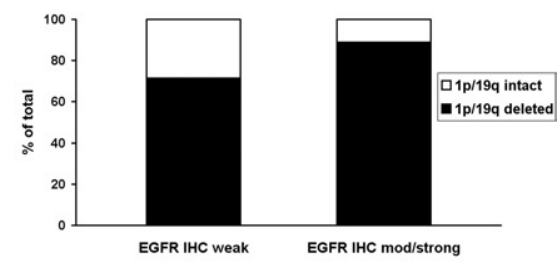

Figure 5. Survival by EGFR IHC in grade III oligodendrogliomas. A: Grade III oligodendrogliomas show worse survival with weak EGFR IHC intensity. $P=0.03$. See Table 3 for specific intergroup $P$ values. B: After combining moderate (mod) and strong EGFR cases into one category, then stratifying by $1 \mathrm{p} / 19 \mathrm{q}$ status, cases with $1 \mathrm{p} / 19 \mathrm{q}$ codeletion had prolonged survival only if EGFR staining was moderate or strong. $P=0.04$ overall, ${ }^{*} P=0.01$ versus EGFR weak, $1 \mathrm{p} / 19 \mathrm{q}$ codeleted tumors. C: There was no significant difference in the likelihood of $1 \mathrm{p} / 19 \mathrm{q}$ codeletion between anaplastic oligodendrogliomas that were strong for EGFR versus those with weaker staining. $P=0.27$.

the three grade II astrocytomas that had EGFR amplification was significantly shorter than similar-appearing tumors lacking amplification (see Supplemental Table S1 at http://ajp.amjpathol.org), suggesting that they should not have been called grade II astrocytomas in the first place.

In our cohort, the degree of EGFR expression had no correlation with survival in grade II or III astrocytic tumors (Figure 3, Table 3), and the survival curves in the GBM set showed no logical pattern, even though the moderately staining group showed a slightly shorter survival than the strongly staining tumors (Figure 3D). These results match the data from most of the smaller studies ${ }^{9-14}$ and confirm that, in astrocytic tumors, EGFR IHC by itself is not a useful prognostic marker.

Regarding EGFR in oligodendroglial tumors, however, a prior retrospective study ${ }^{22}$ of mRNA in 17 grade ॥ oligodendrogliomas showed two distinct expression profiles; the profile that included increased EGFR expression showed worse overall survival than the profile that did not have increased EGFR. Our current study verified this finding in a completely new, larger, prospective cohort of grade II oligodendrogliomas, using a different method to assess expression. Of particular interest is that strong EGFR immunostaining correlated with shorter survival in our cohort even after adjusting for $1 \mathrm{p} / 19 \mathrm{q}$ status (Figure 4). Another study ${ }^{23}$ of 32 oligodendrogliomas did not find a link between EGFR IHC and outcome, although in those cases, only weak expression was ever seen, perhaps suggesting different immunostaining protocols. Indeed, most other studies ${ }^{24,25}$ have shown a wider range of EGFR expression in oligodendroglial tumors, generally increasing with WHO grade.

In GBMs, increased EGFR immunostaining was linked to Akt activation, which in turn trended toward shorter survival. ${ }^{26}$ The previously mentioned mRNA study showed that the grade II oligodendrogliomas with worse outcome had increased EGFR expression, as well as up-regulation of other genes associated with aggressive behavior, such as PPA2 and down-regulation of antitumor-related immune genes, such as HLA class II histocompatibility antigen. The same group also demonstrated increased EGFR expression in a recurrent tumor compared with the original from the same patient, suggesting that EGFR up-regulation may contribute to oligodendroglioma progression. Therefore, grade II oligoden- drogliomas with increased EGFR expression on initial biopsy may simply be more advanced compared with oligodendrogliomas that appear similar histologically but have lower EGFR expression. ${ }^{22}$

The correlation between increased EGFR expression and longer survival in grade III anaplastic oligodendroglial tumors, the exact opposite of what was seen in their grade II counterparts (Figure 5), is more difficult to explain from a biological perspective, considering the prooncogenic effects of EGFR. Yet this also has precedence in the literature, because a recent retrospective mRNA analysis showed the same favorable effect of increased EGFR expression in 39 oligodendroglial tumors, although in that study, ${ }^{27} 12$ grade $\mathrm{II}$ and 27 grade III tumors were pooled together and $1 \mathrm{p} / 19 \mathrm{q}$ data were not available. Judging from our current data, the anaplastic oligodendrogliomas in that pooled cohort may have masked an adverse impact of EGFR in their grade II oligodendrogliomas. The association of EGFR with outcome in grade III oligodendrogliomas cannot be explained by $1 \mathrm{p} / 19 \mathrm{q}$ status, for there was no significant association between EGFR IHC score and 1p/19q status (Figure 5C). In addition, even $1 p / 19 q$ codeleted anaplastic oligodendrogliomas had short survival if EGFR expression was weak (Figure 5B).

These data suggest that the effect of high EGFR in oligodendrogliomas depends greatly on tumor grade, questioning whether there are any differences in expression profiles between the two grades that might account for this. For example, Akt activation can trigger matrix metalloproteinase expression in a p300-dependent mechanism, ${ }^{28}$ yet p300 expression is lower in grade III versus grade II oligodendrogliomas. ${ }^{29}$ Alternatively, excessive oncogene signaling can sometimes inhibit tumor growth via induction of compensatory antiproliferative mechanisms, such as cell senescence. ${ }^{30}$ Perhaps many grade III oligodendrogliomas, already having achieved robust proliferation and invasiveness, cannot respond to additional EGFR in the same way as a less aggressive tumor.

In summary, these data indicate that EGFR IHC is useful in the workup of infiltrative gliomas. Moderate-tostrong EGFR immunostaining may prompt the additional testing of EGFR amplification by FISH, particularly in situations in which the presence of amplification could change the diagnosis (eg, in a tumor that histologically is a grade II astrocytoma). Although EGFR IHC is not helpful 
in modifying the prognosis of astrocytic tumors, it does appear to be useful for the refinement of expected survival in patients with oligodendroglial tumors, even after considering $1 \mathrm{p} / 19 \mathrm{q}$ status. However, in this latter situation the effects of EGFR depend greatly on the WHO grade of the tumor, because it has an adverse impact on survival in grade II oligodendrogliomas but a favorable impact in grade III oligodendrogliomas. Therefore, this study suggests that a rigorous, widely accepted protocol for EGFR immunostaining, evaluation, and reporting (analogous to Her2/neu in breast cancer) may be desirable if this marker is to become part of the routine workup of gliomas, particularly oligodendrogliomas.

\section{Acknowledgments}

We thank the In Situ Hybridization Laboratory personnel at the University of Pittsburgh for their assistance in generating these data.

\section{References}

1. McLendon RE, Turner K, Perkinson K, Rich J: Second messenger systems in human gliomas. Arch Pathol Lab Med 2007, 131:15851590

2. Hatanpaa KJ, Burma S, Zhao D, Habib AA: Epidermal growth factor receptor in glioma: signal transduction, neuropathology, imaging, and radioresistance. Neoplasia 2010, 12:675-684

3. Horbinski C: Practical molecular diagnostics in neuropathology: making a tough job a little easier. Semin Diagn Pathol 2010, 27:105-113

4. Martin V, Mazzucchelli L, Frattini M: An overview of the epiderma growth factor receptor fluorescence in situ hybridisation challenge in tumour pathology. J Clin Pathol 2009, 62:314-324

5. Perry A, Aldape KD, George DH, Burger PC: Small cell astrocytoma: an aggressive variant that is clinicopathologically and genetically distinct from anaplastic oligodendroglioma. Cancer 2004, 101:2318-2326

6. Kouwenhoven MC, Gorlia T, Kros JM, Ibdaih A, Brandes AA, Bromberg JE, Mokhtari K, van Duinen SG, Teepen JL, Wesseling P, Vandenbos F, Grisold W, Sipos L, Mirimanoff R, Vecht CJ, Allgeier A, Lacombe $\mathrm{D}$, van den Bent $\mathrm{MJ}$ : Molecular analysis of anaplastic oligodendroglial tumors in a prospective randomized study: a report from EORTC study 26951. Neuro Oncol 2009, 11:737-746

7. Ronellenfitsch MW, Steinbach JP, Wick W: Epidermal growth factor receptor and mammalian target of rapamycin as therapeutic targets in malignant glioma: current clinical status and perspectives. Target Onco 2010, 5:183-191

8. De Witt Hamer PC: Small molecule kinase inhibitors in glioblastoma: a systematic review of clinical studies. Neuro Oncol 2010, 12:304-316

9. Chakravarti A, Seiferheld W, Tu X, Wang H, Zhang HZ, Ang KK, Hammond E, Curran W Jr, Mehta M: Immunohistochemically determined total epidermal growth factor receptor levels not of prognostic value in newly diagnosed glioblastoma multiforme: report from the Radiation Therapy Oncology Group. Int J Radiat Oncol Biol Phys 2005, 62:318-327

10. Layfield LJ, Willmore C, Tripp S, Jones C, Jensen RL: Epidermal growth factor receptor gene amplification and protein expression in glioblastoma multiforme: prognostic significance and relationship to other prognostic factors. Appl Immunohistochem Mol Morphol 2006 , 14:91-96

11. Miracco C, De Santi MM, Luzi $P$, Lalinga AV, Laurini L, De Nisi MC, Angeloni G, Brogi M, Cardone C, Carducci A, Arcuri F, Tosi P, Rubino G, Pirtoli L: In situ detection of telomeres by fluorescence in situ hybridization and telomerase activity in glioblastoma multiforme: correlation with p53 status, EGFR, c-myc, MIB1, and Topoisomerase Ilalpha protein expression. Int J Oncol 2003, 23:1529-1535

12. Waha A, Baumann A, Wolf HK, Fimmers R, Neumann J, Kindermann D, Astrahantseff K, Blumcke I, von Deimling A, Schlegel U: Lack of prognostic relevance of alterations in the epidermal growth factor receptor-transforming growth factor-alpha pathway in human astrocytic gliomas. J Neurosurg 1996, 85:634-641

13. Muracciole X, Romain S, Dufour H, Palmari J, Chinot O, Ouafik L, Grisoli F, Branger DF, Martin PM: PAI-1 and EGFR expression in adult glioma tumors: toward a molecular prognostic classification. Int J Radiat Oncol Biol Phys 2002, 52:592-598

14. Etienne MC, Formento JL, Lebrun-Frenay C, Gioanni J, Chatel M, Paquis P, Bernard C, Courdi A, Bensadoun RJ, Pignol JP, Francoual M, Grellier P, Frenay M, Milano G: Epidermal growth factor receptor and labeling index are independent prognostic factors in glial tumor outcome. Clin Cancer Res 1998, 4:2383-2390

15. Horbinski C, Hamilton RL, Nikiforov Y, Pollack IF: Association of molecular alterations, including BRAF, with biology and outcome in pilocytic astrocytomas. Acta Neuropathol 2010, 119:641-649

16. Ohgaki $\mathrm{H}$, Kleihues P: Population-based studies on incidence, survival rates, and genetic alterations in astrocytic and oligodendroglial gliomas. J Neuropathol Exp Neurol 2005, 64:479-489

17. Guillaudeau A, Durand K, Pommepuy I, Robert S, Chaunavel A, Lacorre S, DeArmas R, Bourtoumieux S, El Demery M, Moreau JJ, Labrousse F: Determination of EGFR status in gliomas: usefulness of immunohistochemistry and fluorescent in situ hybridization. Appl Immunohistochem Mol Morphol 2009, 17:220-226

18. Fischer I, de la Cruz C, Rivera AL, Aldape K: Utility of chromogenic in situ hybridization (CISH) for detection of EGFR amplification in glioblastoma: comparison with fluorescence in situ hybridization (FISH). Diagn Mol Pathol 2008, 17:227-230

19. Kim B, Myung JK, Seo JH, Park CK, Paek SH, Kim DG, Jung HW, Park $\mathrm{SH}$ : The clinicopathologic values of the molecules associated with the main pathogenesis of the glioblastoma. J Neurol Sci 2010, 294:112-118

20. Pollack IF, Hamilton RL, James CD, Finkelstein SD, Burnham J, Yates AJ, Holmes EJ, Zhou T, Finlay JL: Rarity of PTEN deletions and EGFR amplification in malignant gliomas of childhood: results from the Children's Cancer Group 945 cohort. J Neurosurg 2006, 105:418-424

21. Ohgaki H, Dessen P, Jourde B, Horstmann S, Nishikawa T, Di Patre PL, Burkhard C, Schuler D, Probst-Hensch NM, Maiorka PC, Baeza N, Pisani P, Yonekawa Y, Yasargil MG, Lutolf UM, Kleihues P: Genetic pathways to glioblastoma: a population-based study. Cancer Res 2004, 64:6892-6899

22. Huang H, Okamoto $\mathrm{Y}$, Yokoo H, Heppner FL, Vital A, Fevre-Montange M, Jouvet A, Yonekawa Y, Lazaridis EN, Kleihues P, Ohgaki H: Gene expression profiling and subgroup identification of oligodendrogliomas. Oncogene 2004, 23:6012-6022

23. Broholm H, Bols B, Heegaard S, Braendstrup O: Immunohistochemical investigation of p53 and EGFR expression of oligodendrogliomas. Clin Neuropathol 1999, 18:176-180

24. Reifenberger J, Reifenberger G, Ichimura K, Schmidt EE, Wechsler W. Collins VP: Epidermal growth factor receptor expression in oligodendroglial tumors. Am J Pathol 1996, 149:29-35

25. McLendon RE, Wikstrand CJ, Matthews MR, Al-Baradei R, Bigner SH, Bigner DD: Glioma-associated antigen expression in oligodendroglial neoplasms: tenascin and epidermal growth factor receptor. J Histochem Cytochem 2000, 48:1103-1110

26. Mizoguchi M, Betensky RA, Batchelor TT, Bernay DC, Louis DN, Nutt CL: Activation of STAT3, MAPK, and AKT in malignant astrocytic gliomas: correlation with EGFR status, tumor grade, and survival. J Neuropathol Exp Neurol 2006, 65:1181-1188

27. Zhou Y-H, Hess KR, Raj VR, Yu L, Liu L, Yung AWK, Linskey ME: Establishment of prognostic models for astrocytic and oligodendroglial brain tumors with standardized quantification of marker gene expression and clinical variables. Biomarker Insights 2010, 5:153-168

28. Lee CW, Lin CC, Lin WN, Liang KC, Luo SF, Wu CB, Wang SW, Yang CM: TNF-alpha induces MMP-9 expression via activation of Srcl EGFR, PDGFR/PI3K/Akt cascade and promotion of NF-kappaB/p300 binding in human tracheal smooth muscle cells. Am J Physiol Lung Cell Mol Physiol 2007, 292:L799-L812

29. Tews B, Felsberg J, Hartmann C, Kunitz A, Hahn M, Toedt G, Neben K, Hummerich L, von Deimling A, Reifenberger G, Lichter P: Identification of novel oligodendroglioma-associated candidate tumor suppressor genes in $1 p 36$ and 19q13 using microarray-based expression profiling. Int J Cancer 2006, 119:792-800

30. Hanahan D, Weinberg RA: Hallmarks of cancer: the next generation. Cell 2011, 144:646-674 